\title{
Nuevo mapa conceptual de la investigación científica en ciencias de los alimentos basado en técnicas cienciométricas en España (2003-2014)
}

New Scientometric-based knowledge map of Food Science research in Spain (2003-2014)

Jesús BlÁZquez-Ruiz (1), Vicente P. Guerrero-Bote (2), Félix MoyA-Anegón (2)

(1) Universidad de Extremadura, Departamento de Información y Comunicación, España, jblazques@alumnos.unex.es (2) Universidad de Extremadura, Departamento de Información y Comunicación, España, guerrero@unex.es

(3) CSIC, CCHS, IPP, Grupo Scimago, España, felix.moya@scimago.es

\section{Resumen}

Se realiza un análisis de palabras clave de la investigación en Ciencia de los Alimentos en España con el objetivo de descubrir sus líneas de investigación más activas en el periodo 2003-2014, comparando la evolución con la investigación mundial en la disciplina. Para ello se descargaron todos los registros bibliográficos de Scopus y se resolvieron los problemas obvios de sinonimia y se seleccionaron las más destacadas. Estas palabras clave fueron agrupadas en campos y subcampos utilizando técnicas del análisis de copalabras para toda la producción mundial y así tener una referencia para el estudio a nivel nacional. Se comprueba que España es una gran potencia en la investigación en Ciencias de los Alimentos, no tanto por su volumen de producción científica, que también, sino más bien por la calidad de ésta. España destaca especialmente en los campos de "Conservación de alimentos y vida útil" y "Antioxidantes".

Palabras clave: Análisis temático. Análisis de copalabras. Cienciometría. Ciencia de los alimentos. Estructura de la ciencia de los alimentos. Frentes de investigación. España.

\section{Introducción}

Una de las virtudes de la ciencia es dar respuesta a las necesidades que se van generando en el mundo y en la sociedad. En la actualidad nos encontramos ante un problema de carácter primordial como es alimentar a todas las personas del planeta sabiendo que cada día somos más, y, ante este problema, la ciencia ha respondido con la Ciencia de los Alimentos (CA en adelante). La CA es la disciplina en que se estudia "la estructura física, biológica y química de los alimentos; las causas del deterioro de los alimentos; y los conceptos subyacentes del procesado de alimentos" (IFT, 2016).

Si tenemos en cuenta que a fecha de 2015 éramos unos 7.300 millones de personas, en 2030 seremos unos 8.500 millones, en 2050 unos 9.700 millones y en 2100 unos 11.200

\begin{abstract}
A keyword analysis of the research in Food Science in Spain is carried out with the objective of discovering the most active lines of research in the period 2003-2014, comparing its evolution with the world research in the discipline. For this purpose, all the bibliographical records of Scopus were downloaded and their keywords were processed to solve the obvious problems of synonymy and to limit the study to those most frequently used. These keywords were grouped in fields and subfields using co-word analysis techniques. This was done with the world production so that we could have a reference for the study at the national level. It is verified that Spain is a great power in scientific research in the field of Food Science, not only by the volume of its scientific production, but also by its quality. Spain stands out especially in the fields of "Food Preservation and Shelf Life" and "Antioxidants".
\end{abstract}

Keywords: Food Science. Thematic analysis. Food Science structure. Co-word analysis. Scientometrics. Research fronts. Spain.

millones (Wiley-Blackwell, 2015) o tal vez más, hasta unos 13.200 millones (Gerland et al., 2014), entonces nos damos cuenta que no se trata de una cuestión baladí el hecho de pensar en la CA como algo realmente necesario para el presente y el futuro. De hecho, en estos momentos, la CA está generando una gran cantidad de trabajos y estudios sobre su temática a nivel internacional. Dos de ellos son el de Guerrero-Bote, Olmeda-Gómez y Moya-Anegón, (2016a) el cual caracteriza la actividad investigadora de instituciones internacionales dedicadas a la CA; y el de Guerrero-Bote y MoyaAnegón de 2015 que entre sus conclusiones apunta que "la producción científica en CA ha crecido a gran ritmo en la última década, a un ritmo superior al resto de la producción científica mundial", siendo España uno de los tres países que mayor producción científica genera en este área, junto con China y Estados Unidos. 
Sabiendo que en España las CA son pues una disciplina muy estudiada, creemos necesario realizar un estudio cienciométrico que, apelando a su papel de evaluación (Mingers y Leydesdorff, 2015), identifique cómo se desarroIla la actividad científica investigadora en España en relación con el mundo, y nos permita saber en qué aspectos tenemos que mejorar y cuáles tenemos que reforzar. Hay otros estudios que abordan el tema de las CA en España desde una perspectiva cienciométrica, pero son pocos. Algunos ejemplos son el estudio de Chinchilla-Rodríguez y Olmeda-Gómez (2010) quienes, a través de un análisis multinivel, pretenden "caracterizar la generación de conocimiento visible internacionalmente en el campo de la agroalimentación en España durante los últimos quince años a partir del análisis de los resultados de investigación en forma de publicaciones científicas"; y el de Guerrero-Bote, Olmeda-Gómez y Moya-Anegón, en un trabajo de 2016, que buscan conocer el grado de reconocimiento internacional que tienen las producciones científicas llevadas a cabo por centros de investigación españoles.

En este trabajo nos marcamos como objetivo principal analizar temáticamente la producción científica española en CA para poder compararla con la mundial (Blázquez-Ruiz, Guerrero-Bote y Moya-Anegón, 2016). Además, para llevar a cabo adecuadamente este objetivo principal, nos hemos marcado también el objetivo específico de generar un mapa de copalabras que represente la organización de los 5 campos generales que vertebran la estructura de las CA, según el trabajo de Blázquez-Ruiz, GuerreroBote y Moya-Anegón (2016).

Estos objetivos nos conducen a hacernos las siguientes preguntas de investigación:

- ¿Cómo evoluciona la producción española en CA en cada área o campo temático?

- ¿Cómo se relacionan las 5 áreas temáticas generales entre sí?

- ¿Cómo de cohesionadas internamente aparecen las 5 áreas?

- ¿Qué temas son los más centrales y los más especializados?

- ¿Cuenta España con un impacto científico destacado en cada área de investigación?

- ¿Cómo son las palabras clave de moda en la investigación en CA en España en el periodo de estudio?

\section{Metodología}

Este trabajo utiliza como base para su desarroIlo una colección de palabras clave que, extraídas de la base de datos Scopus, ha permitido realizar las distintas inferencias para cumplir los objetivos antes propuestos.

Scopus es una de las bases de datos bibliográficas que mayor cantidad de revistas científicas recoge y ha sido utilizada en gran cantidad de investigaciones cienciométricas. Además, también ha sido estudiada en sí misma y en comparación con otras bases de datos similares. El trabajo de Falagas et al. (2008) que compara las bases de datos PubMed, Scopus, Web of Science(WoS) y Google Scholar concluye que Scopus cuenta con un espectro más amplio de revistas que PubMed y WoS y que su análisis de citas es más rápido e incluye más artículos que el análisis de citas de WoS (de Google Scholar destaca sus insuficiencias en la búsqueda científica y el hecho de que mucha información acerca de su contenido sea desconocida). También Granda-Orive et al. (2013) realiza otro de estos estudios en el que compara la base de datos de Scopus con la de Web of Science en el tema concreto del tabaquismo y entre sus conclusiones nos dice que Scopus "presentó un mayor número de revistas, de trabajos, y de firmas". Por último, mencionamos también un trabajo de Moya-Anegón et al. de 2006, que estudia la cobertura de Scopus y que determina que "en números redondos Scopus representa el $50 \%$ del universo de revistas peerreviewed, mientras que ISI-WoS solo el 25\%". Por todos estos estudios y por las características propias de nuestro trabajo hemos decidido trabajar con Scopus en lugar de con cualquier otra base de datos.

Las palabras clave que permite descargar Scopus son de dos tipos, las "Author Keywords" (asignadas por el propio autor) y las "Index Keywords" (asignadas por indizadores profesionales). Nosotros hemos descargado las de autor por ser más numerosas y representativas del contenido de los documentos. En esta investigación hemos elegido descargar las "Author Keywords" de trabajos publicados en todas las revistas incluidas en la "specific subject area" de Scopus, "Food Science", en el periodo 20032014 para hacer el análisis de las mismas. Fueron descargadas un total de 230.007 palabras clave, de 184.801 artículos. En investigaciones similares, la metodología a la hora de descargar las keywords puede ser diferente, por ejemplo, en el trabajo de Romo-Fernández, GuerreroBote y Moya-Anegón, de 2013, en el cual se hace un análisis de palabras clave para revelar 
patrones en el campo de las energías renovables, solamente se descargan las palabras clave de una revista del área en cuestión.

Con las palabras clave descargadas, el siguiente paso consistió en normalizarlas. Para ello se creó una base de datos que, gracias a las distancias de Levenshtein y de DamerauLevenshtein, permitió localizar palabras parecidas y unificarlas en caso de ser la misma. La distancia de Levenshtein (Levenshtein, 1966) cuenta el número mínimo de operaciones que hacen falta para transformar una cadena de caracteres en otra, entendiendo por operaciones la inserción, la eliminación o la sustitución de un carácter. Por otra parte, la distancia de Damerau-Levenshtein (Damerau, 1964) incluye las 3 operaciones anteriores y además la de transposición, operación que en la distancia de Levenshtein contaría como 2 operaciones (eliminación e inserción). El proceso de normalización consistió básicamente en tres procesos: eliminar signos de puntuación sin significado, unificar singulares y plurales, y agrupar parejas de palabras clave similares, utilizando para ello las distancias de Levenshtein y DamerauLevenshtein, para unificar automáticamente las que tenían una distancia pequeña y una gran parte en común o para revisar manualmente las que no cumplían esa condición.

Hecha la normalización, se generó la red de copalabras que nos permitió agruparlas por clústeres gracias al algoritmo de Noack (2007), el cual se basa en el método de detección de comunidades de Newman y Girvan (2004). Las redes de copalabras constan de nodos (palabras claves) y enlaces que enlazan unos nodos con otros y que aparecen ponderados según el número de documentos en el que aparecen ambos nodos. Concretamente, para generar la red de copalabras de este trabajo se fijó un umbral mínimo de frecuencia de aparición de las palabras clave, en 300 documentos, siendo 297 las palabras clave que cumplían este requisito y las que finalmente formarían la red. Ya con la red generada, y teniendo en cuenta la aparición de España como país colaborador de las investigaciones, se construyó el mapa que nos permitiría representar visualmente los resultados obtenidos. Aunque estas representaciones pueden sufrir sesgos y presentar ciertas carencias, en nuestro caso nos ofrecen la ventaja de permitirnos observar de manera clara cómo se relacionan unas comunidades de nodos con otras, permitiéndonos así extraer más conclusiones y en definitiva analizar con mayor profundidad los datos.

Acudimos a los métodos de detección de comunidades para hacer estas representaciones, los cuales permiten detectar grupos de nodos que se relacionan más entre sí. Para hacer esto, Waltman et al. (2010) proponen una variante ponderada y parametrizada de clustering basada en la modularidad que implementan en el VOSviewer (Van-Eck \& Waltman, 2007). Esté método nos permite variar la resolución de las comunidades permitiendo obtener clasificaciones de diferente granularidad.

En este trabajo también se ha utilizado el software VOSviewer de Van-Eck y Waltman en su última versión para hallar el layout, empleando los parámetros de atracción y repulsión propios del Linlog de Noack (Noack, 2009), porque como afirma Van Eck (2011) "an interesting mapping technique that seems to yield accurate results both at the local and at the global level is the LinLog technique".

También se ha utilizado el algoritmo de bursting de Jon Kleinberg (2003) que detecta cuándo ciertos términos se ponen de moda en un discurso y cuándo terminan apagándose, para aplicarlo a nuestras palabras clave en el periodo 2003-2014. El algoritmo nos devuelve una tabla con las palabras clave que han estado alguna vez de moda, indicándonos la longitud, intensidad y periodo de tiempo de la moda.

Por último hemos utilizado también algunos indicadores de impacto científico para complementar mejor todo el trabajo:

- Ndocc. Número de documentos citables publicados en revistas científicas recogidas en la base de datos de Scopus, es decir, articles, reviews, conference papers y short surveys.

- Citas por documento. Promedio de citas por documento.

- Porcentaje de documentos citados.

- Impacto normalizado. Promedio de la citación normalizada recibida por cada documento. Entendiéndose ésta como la ratio entre la citación recibida por el documento y la citación promedio de los documentos del mismo tipo, año y categoría (Rehn \& Kronman, 2008).

- Porcentaje de Excelencia 10. Porcentaje de documentos que se encuentran dentro del $10 \%$ más citado del mismo año, tipo y categoría (Bornmann, Moya-Anegón \& Leydesdorff, 2012).

- Porcentaje de Excelencia 1. Porcentaje de documentos que se encuentran dentro del $1 \%$ más citado del mismo año, tipo y categoría.

Los datos obtenidos tras realizar todo el proceso metodológico anterior, fueron comparados con los del trabajo de Blázquez-Ruiz, Guerrero-Bote, \& Moya-Anegón (2016) que se corresponden con los datos mundiales. 


\section{Resultados}

En el trabajo previo comentado antes (BlázquezRuiz, Guerrero-Bote y Moya-Anegón, 2016), quedó establecida una estructura temática global de las CA que vamos a utilizar como base temática para nuestro estudio. Esta estructura es una estructura jerárquica de clústeres que fue generada en 2 pasos. En el primero se fijó, en el software VOSviewer, el parámetro de resolución en 1.9 , lo que dio lugar a 18 clústeres o subcampos. En el segundo paso, y para agrupar esos 18 subcampos en campos más generales, se fijó el parámetro min size cluster en 35 y 5 fueron los campos generales resultantes. A continuación se sometió la estructura obtenida a la validación por parte de un experto en Ciencias de los Alimentos con cuya ayuda se etiquetaron todos los campos y subcampos generados, para así dotar a la estructura de una denominación concreta. Esta es la estructura temática con sus 5 niveles generales y sus 18 específicos:

1. Composición de alimentos y nutrición.

1.1. Nutrición y metabolismo.

1.2. Nutrientes y calidad.

1.3. Composición alimentos.

1.4. Composición calidad.

2. Procesado y modificado de alimentos.

2.1. Influencia del procesado en características sensoriales de alimentos.

2.2. Métodos de procesado o tratamiento de alimentos.
2.3. Utilización de microorganismos beneficiosos como cultivos iniciadores en el procesado de alimentos.

2.4. Modificaciones de alimentos durante el procesado y forma de determinar estos cambios.

3. Seguridad alimentaria.

3.1. Microorganismos patógenos en leche y productos lácteos.

3.2. Micotoxinas en cereales y métodos de detección de micotoxinas en alimentos.

3.3. Otros contaminantes de alimentos importantes en seguridad alimentaria (metales pesados, pesticidas). Evaluación del riesgo de estos compuestos.

3.4. Agentes antimicrobianos utilizados en alimentos. Métodos de determinación de estos agentes antimicrobianos.

4. Conservación de alimentos y vida útil.

4.1. Incremento de vida útil en alimentos vegetales durante la conservación. Métodos y modificaciones de calidad.

4.2. Proceso de fermentación en vino y cerveza como método de conservación.

4.3. Parámetros que condicionan conservación de alimentos.

4.4. Métodos de análisis estadístico.

5. Antioxidantes en alimentos.

5.1. Antioxidantes y sus efectos.

5.2. Antioxidantes vegetales.

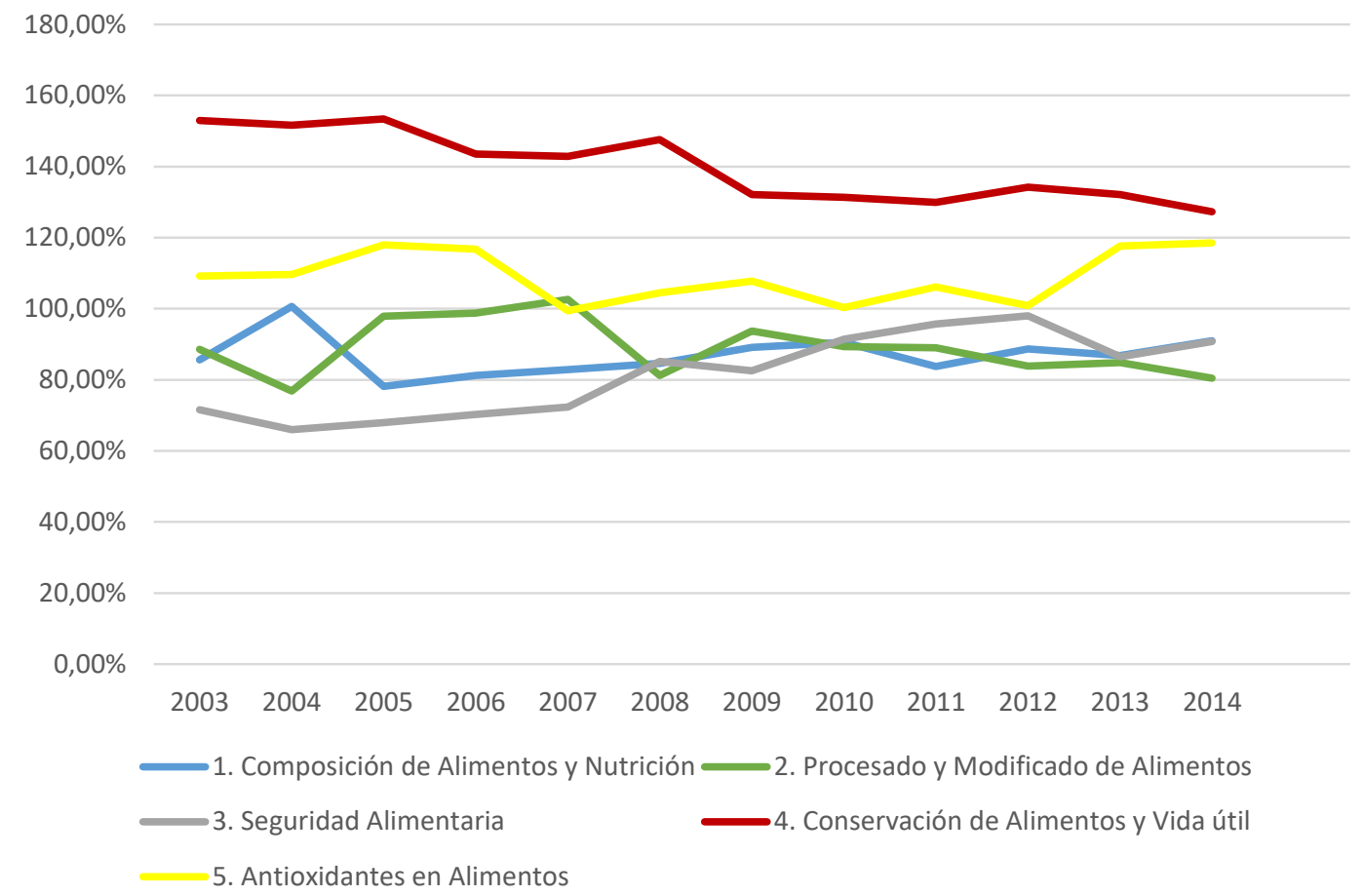

Figura 1. Evolución de la producción relativa respecto al mundo 
La Figura 1 nos muestra la evolución de la producción relativa de la investigación española en CA con respecto al mundo, a través de los cinco campos que conforman la disciplina. Más concretamente, la ratio de producción en cada campo con respecto al total en CA en España, y todo ello frente a la misma ratio a nivel mundial.

Sabiendo que encontrarse en la línea del 100\% del gráfico es equivalente a producir el mismo porcentaje en cada campo, respecto al total en $\mathrm{CA}$, que el conjunto de la producción mundial, todo lo que está por encima del $100 \%$ supera al mundo y viceversa. De este modo, se observa cómo los trabajos españoles que se ocupan de los temas representados por el campo número 4 de las CA, el dedicado a la "Conservación de alimentos y vida útil", suponen, de manera relativa y durante todo el periodo estudiado, una mayor producción que la generada por el conjunto de la investigación mundial. La mayor diferencia positiva para este campo se produce en 2005, donde la producción española supera, siempre hablando de manera relativa, en un $53,45 \%$ a la mundial.

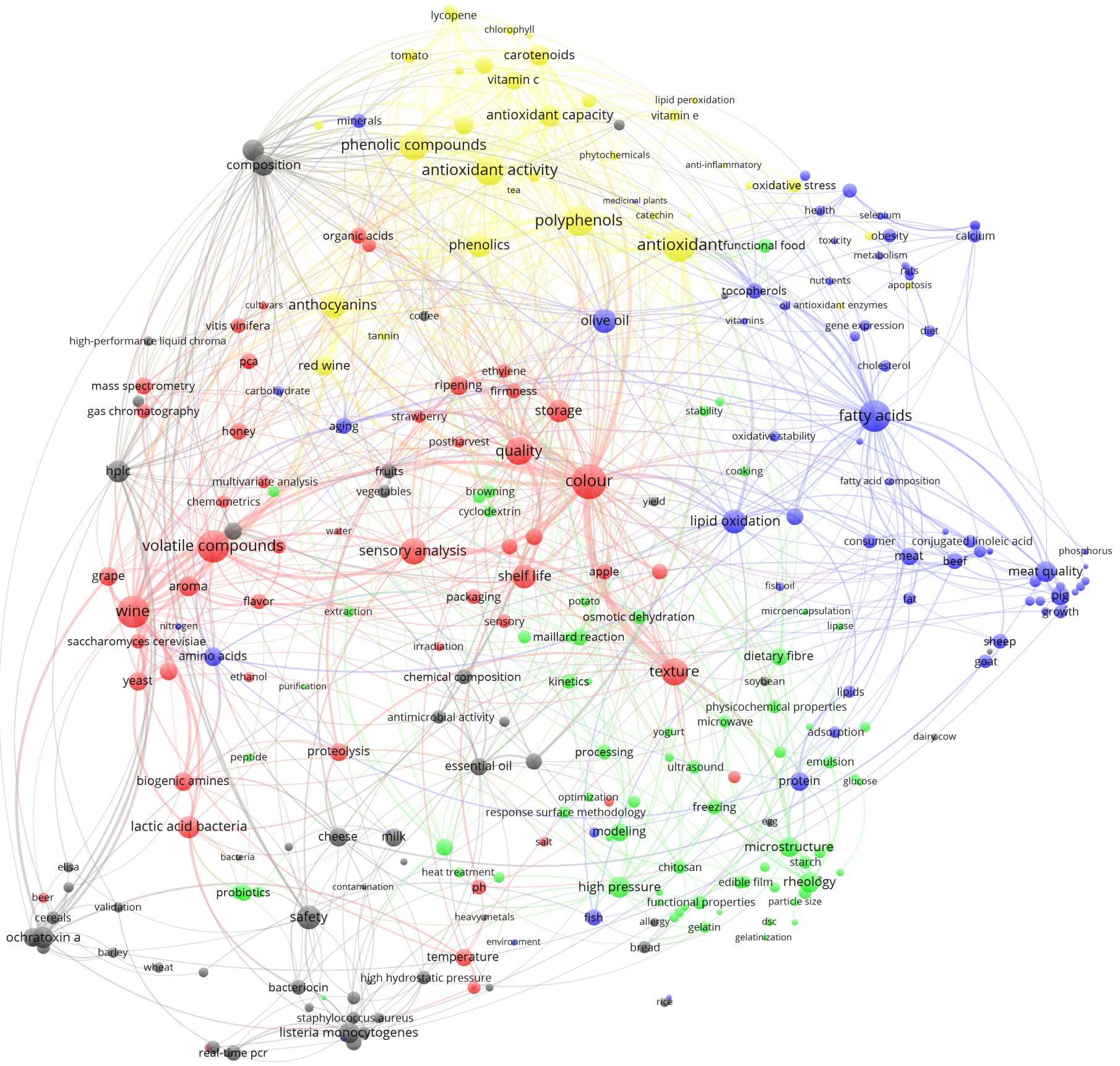

Figura 2. Vista general del mapa de copalabras distinguiendo los 5 clústeres generales 


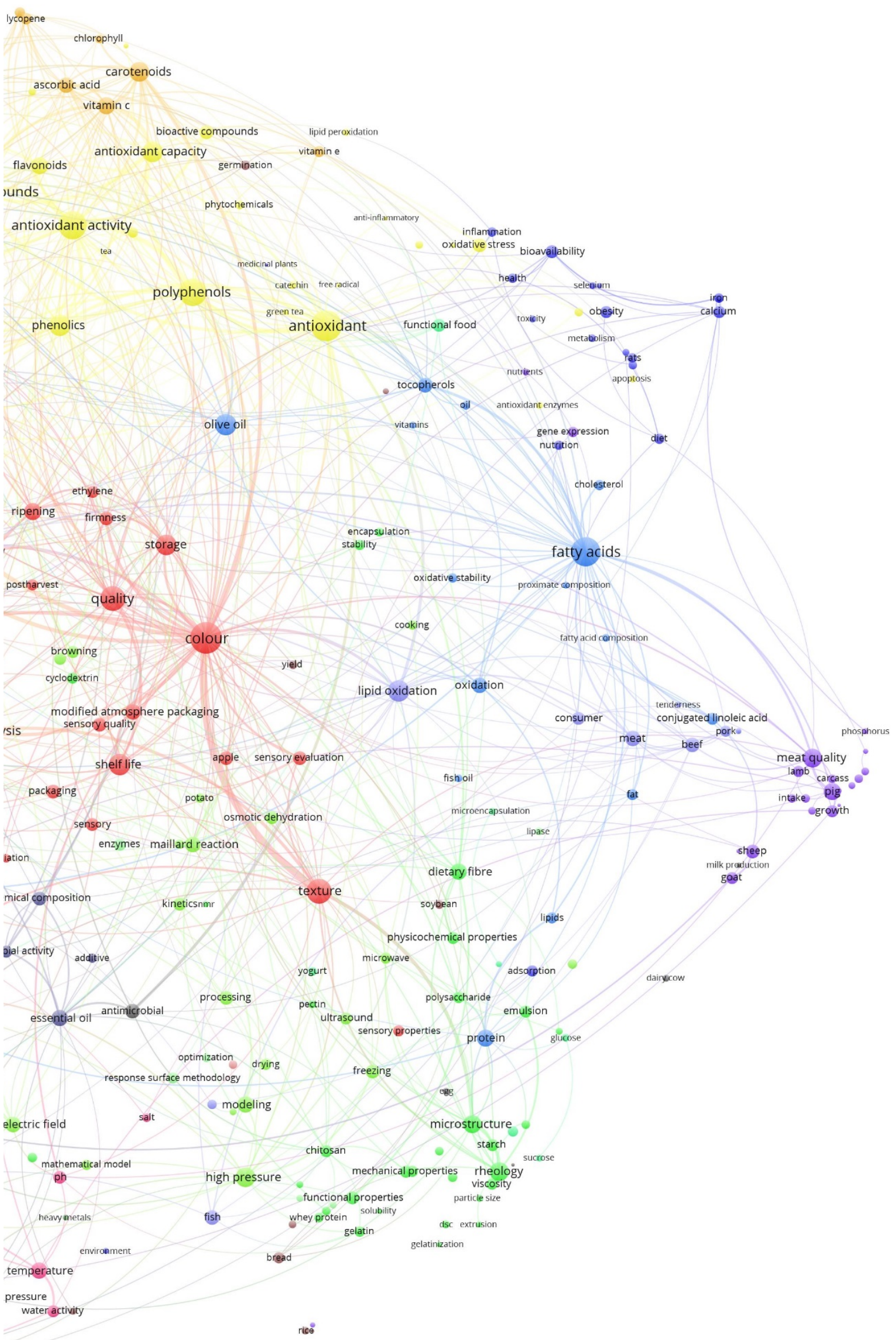

Figura 3. Zoom de la zona Este del mapa de copalabras

Blázquez-Ruiz, Jesús; Guerrero-Bote, Vicente P.; Moya-Anegón, F. Nuevo mapa conceptual de la investigación científica en ciencias de los alimentos basado en técnicas cienciométricas en España (2003-2014). // Ibersid. 11:1 (en.-jun., 2017) 13-31. ISSN 1888-0967. 


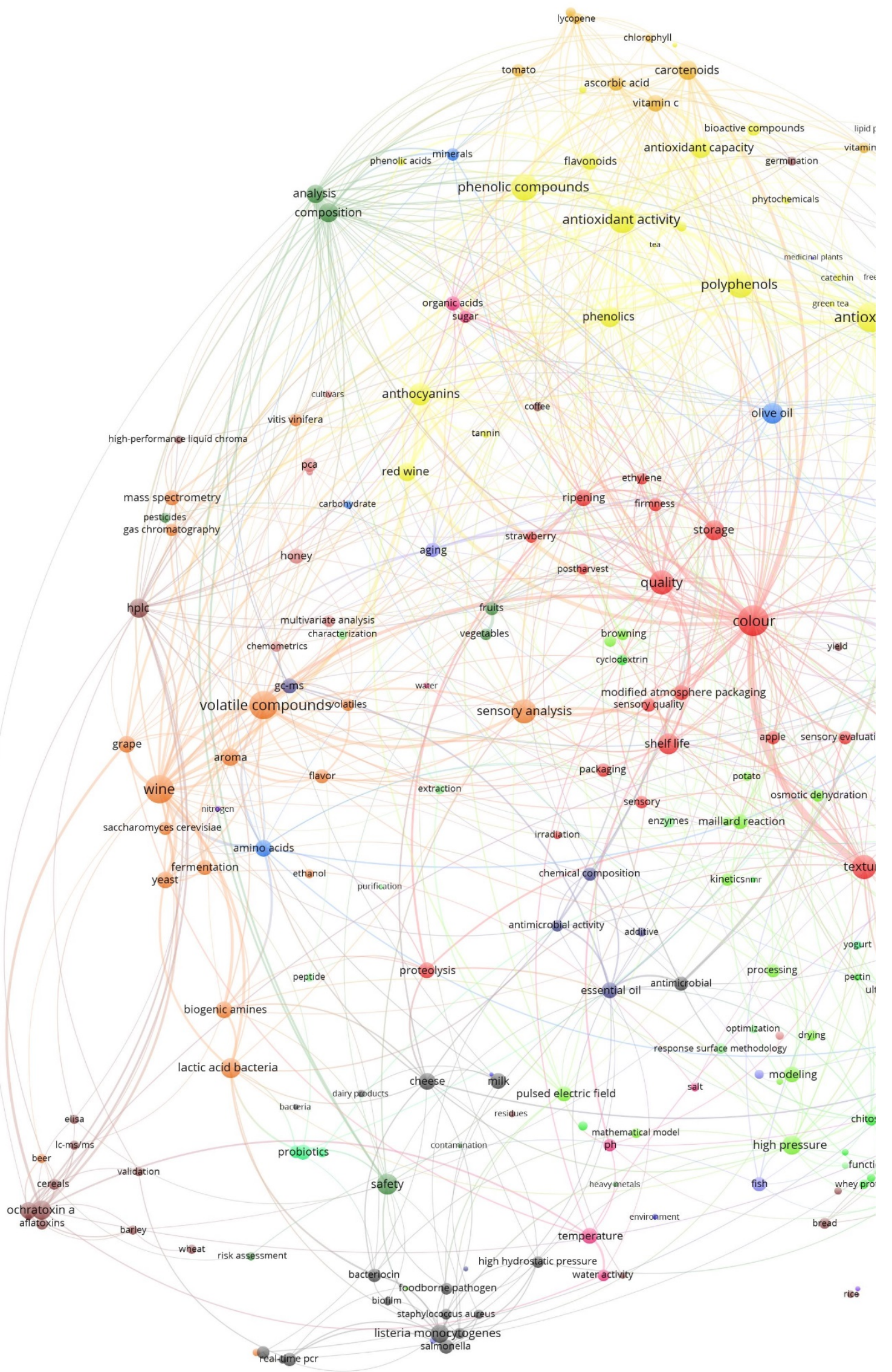

Figura 4. Zoom de la zona Oeste del mapa de copalabras

Blázquez-Ruiz, Jesús; Guerrero-Bote, Vicente P.; Moya-Anegón, F. Nuevo mapa conceptual de la investigación científica en ciencias de los alimentos basado en técnicas cienciométricas en España (2003-2014). // Ibersid. 11:1 (en.-jun., 2017) 13-31. ISSN $1888-0967$. 
Al igual que el campo "Conservación de alimentos y vida útil", el campo 5 dedicado a los "Antioxidantes en alimentos", también supera a la producción mundial durante todo el periodo, exceptuando el año 2007 que se queda a un $0,59 \%$ de igualar esta producción. En esta ocasión las diferencias son algo más bajas que las del campo 4, pero aun así la producción sigue siendo mayor. En el año 2014 se produce la mayor diferencia, superando la producción española a la mundial en un $18,52 \%$.

Como se ve en el gráfico el resto de campos se encuentran por debajo de la barrera del $100 \%$ indicando que generan menor porcentaje que el conjunto mundial en sus respectivas áreas, integrantes todas de las CA. Aun así, hay dos momentos en los que estos campos superan levemente a la producción mundial: en 2004 el campo número 1 , dedicado a la "Composición de alimentos y nutrición", lo hace por un $0,66 \%$ y en 2007 el número 2, dedicado al "Procesado y modificado de alimentos", lo hace por un 2,64\%. El resto de años los campos 1, 2 y 3 están siempre por debajo, estableciéndose la mayor diferencia negativa en 2004, donde el campo 3, dedicado a "Seguridad alimentaria", cae en un $33,95 \%$. Hay que tener en cuenta, cómo se compara el porcentaje de producción dentro de $\mathrm{CA}$, para que unos estén por encima del mundo los otros tienen que estar por debajo. Porque en términos absolutos como podemos ver en el trabajo de 2016 de Guerrero-Bote, OlmedaGómez, \& Moya-Anegón, la producción científica en CA en España en el periodo de estudio tiene un crecimiento sostenido similar al mundial.

La Figura 2 presenta los 5 clústeres de resolución 1 (campos) que se han formado, a través de un mapa de copalabras. En este tipo de mapas, los clústeres se diferencian por colores, variando el tamaño de la etiqueta y el del círculo de sus nodos (palabras clave) según el número de documentos asociados a cada nodo. Así mismo, las figuras 3 y 4 muestran un zoom de las zonas este y oeste respectivamente y diferencian los clústeres de resolución 2 (subcampos) mediante distintas tonalidades del color del clúster de resolución 1 al que pertenecen.

Al buscar cuál es el campo general con mayor cohesión, nos damos cuenta que es el dedicado a los "Antioxidantes en alimentos" (color amariIlo), el que cuenta con una mayor cohesión interna entre sus palabras clave. Este campo se subdivide en otros 2 subcampos y se encuentra en una única zona del mapa, siendo esta una característica esencial que nos permite determinar que es el campo que mayor cohesión interna presenta entre sus nodos, los cuales están bien relacionados entre sí y apenas presentan dispersión y solapamiento con los nodos de otros campos.

El campo dedicado a la "Conservación de alimentos y vida útil" (color rojo), también presenta una muy buena cohesión interna, pero en este caso se observa una división vertical en el mapa, a través del mismo, que establece dos zonas del mismo bien cohesionadas internamente por separado, correspondiéndose cada una con un subcampo del mismo. Concretamente, la zona más al oeste se corresponde con el dedicado al "Proceso de fermentación en vino y cerveza como método de conservación"; y la zona más al centro se corresponde con el dedicado al "Incremento de vida útil en alimentos vegetales durante la conservación. Métodos y modificaciones de calidad". Aparte de estos dos subcampos, los otros dos que conforman la "Conservación de alimentos y vida útil" se encuentran en la zona oeste del mapa pero mostrando una mayor dispersión entre sus nodos. En el mapa de densidad (Figura 6, en la página siguiente) se observa muy bien la división vertical de la que hablamos.

En cuanto a la centralidad, a simple vista se observa que es el campo número 4 el que mayor grado de centralidad presenta. Como comentábamos antes, este campo aparece dividido en 2 zonas, pero no cabe duda que una de ellas, la relativa al "Incremento de vida útil en alimentos vegetales durante la conservación. Métodos y modificaciones de calidad", ocupa el centro del mapa. Además, si nos fijamos nuevamente en el mapa de densidad, comprobamos que esta zona está muy destacada en él.

Ahora bien, cuando comparamos este mapa con el generado a nivel global (Figura 5) nos damos cuenta que hay varias diferencias. Comentábamos antes que el campo de los "Antioxidantes en alimentos" era el que mayor cohesión presentaba y esto mismo pasa en el caso mundial, estando aún más cohesionado que en el mapa de España. La principal diferencia la encontramos en lo referente a la centralidad, mientras que en España hay una ocupación bien definida del centro del mapa, en el caso mundial no ocurre lo mismo y de hecho no se puede decir que haya ningún campo que destaque por su centralidad.

En lo que respecta a las relaciones entre campos y subcampos, estas se producen cuando los nodos que los forman comparten un mismo documento, es decir, cuando en un documento aparecen palabras clave o nodos de 2 campos o subcampos distintos. Mientras en más documentos aparezcan dos nodos concretos, mayor será su tamaño en la representación de los mapas y más fuerte será la relación entre ambos. 


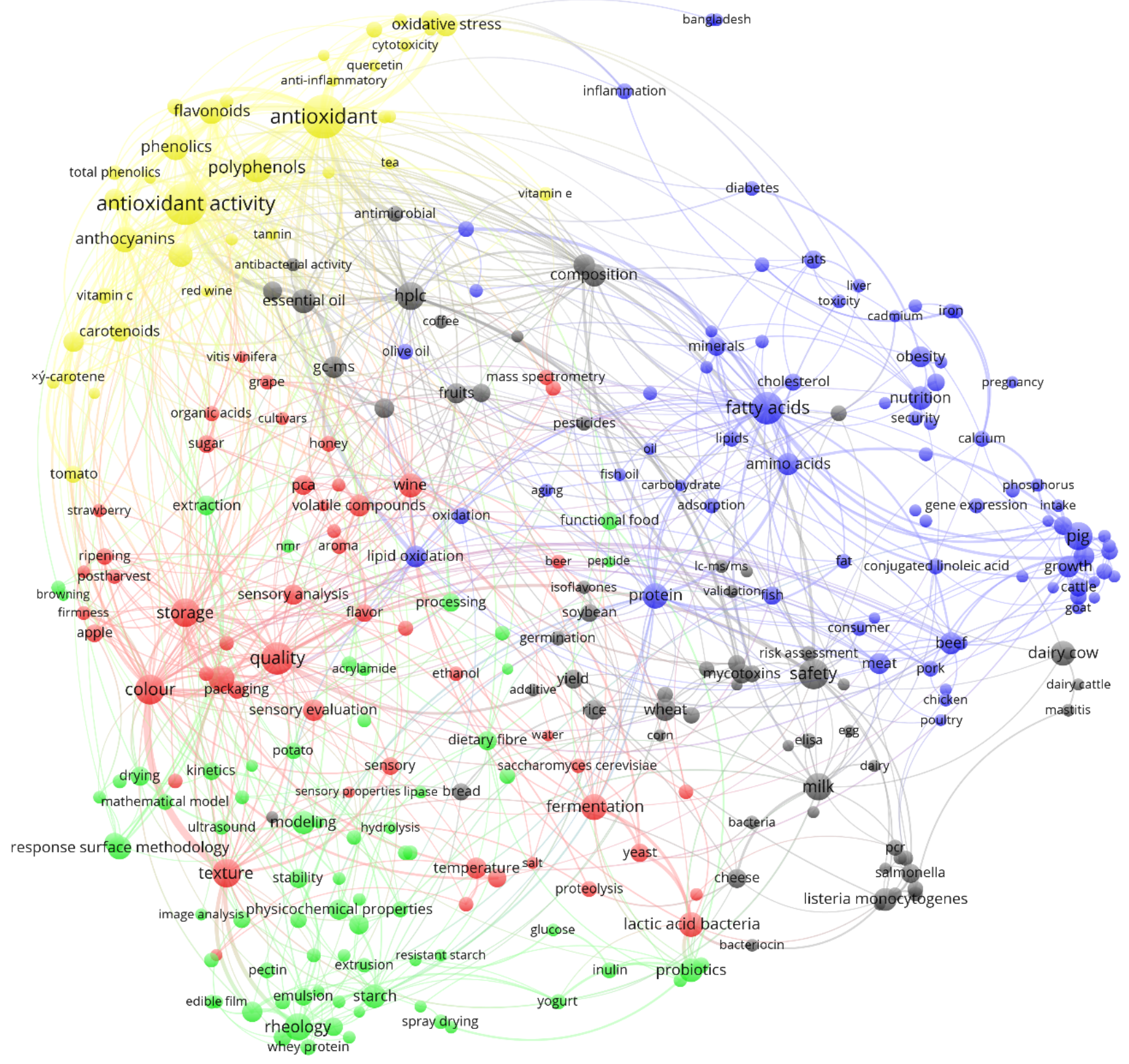

Figura 5. Vista general del mapa de copalabras mundial distinguiendo los 5 clústeres generales

Sabiendo esto, determinar qué campos están mejor relacionados con otros es posible atendiendo a los nodos mejor relacionados. Observando las relaciones que se establecen en cada uno de los dos casos, comprobamos que los nodos que configuran los 5 campos, como es lógico, mantienen las relaciones más significativas con nodos de su mismo campo. Pero dejando a un lado esto y sabiendo que todos los campos mantienen relaciones con todos, aquí vamos a destacar las relaciones más fuertes y es donde observaremos las principales diferencias entre los dos casos.
- El campo dedicado a la "Composición de alimentos y nutrición" en el caso de España, situado principalmente al este del mapa, mantiene fuertes conexiones con los campos relativos a la "Seguridad alimentaria" y a la "Conservación de alimentos y vida útil". En el mapa del mundo las relaciones más fuertes son establecidas únicamente con el último.

- En ambos casos en el campo dedicado al "Procesado y modificado de alimentos" se observa una leve dispersión de sus nodos y pocas relaciones externas. Además, de un primer vistazo es el que presenta los nodos más pequeños, dándonos a entender que puede 
ser el campo con menor cantidad de documentos asociados a cada uno de sus nodos.

- El campo de "Seguridad alimentaria" también en ambos casos se encuentra muy disperso por el mapa aunque en el caso español está más concentrado en la zona oeste del mismo. Mantiene buenas relaciones, en mayor o menor medida, con el resto de campos, excepto con el del "Procesado y modificado de alimentos" en el caso de España.

- Es en el campo dedicado a la "Conservación de alimentos y vida útil" donde encontramos las diferencias más destacadas, entre uno y otro caso, en lo que a relaciones con otros clústeres se refiere. Mientras que en España mantiene fuertes relaciones con los "Antioxidante en alimentos" y en menor medida con el "Procesado y modificado de alimentos", en el mundo estas relaciones las mantiene con la "Composición de alimentos y nutrición".

- Por último, el campo de los antioxidantes también presenta diferencias. Mientras que en el caso español la buena relación con la "Conservación de alimentos y vida útil" es recíproca, en el mundo solo mantiene fuertes relaciones con el tema de la "Seguridad alimentaria" y además únicamente a través de sus dos nodos principales. En ambos casos vemos que está bien localizado en una zona específica de los dos mapas.

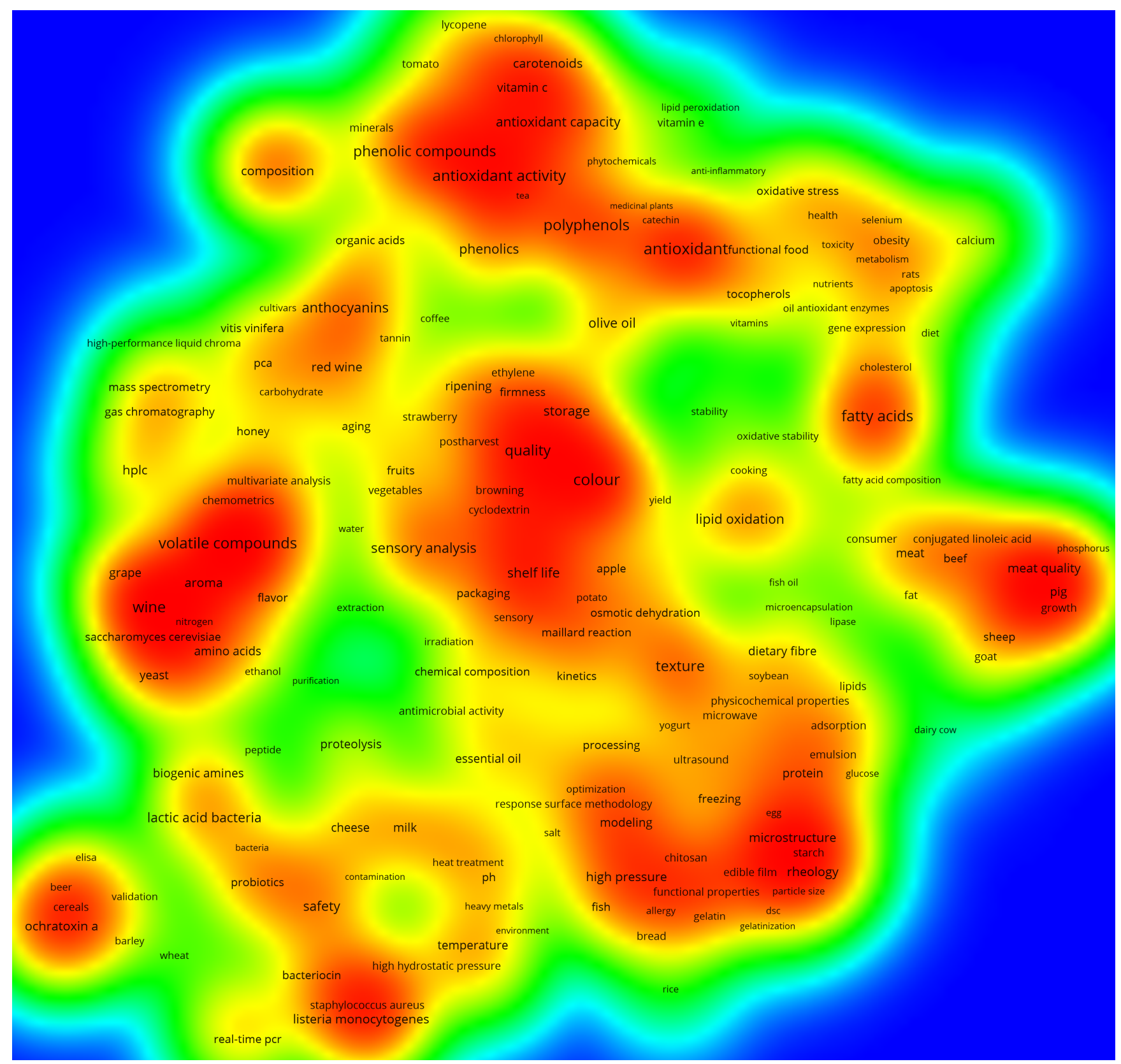

Figura 6. Vista de densidad del mapa general de copalabras

Blázquez-Ruiz, Jesús; Guerrero-Bote, Vicente P.; Moya-Anegón, F. Nuevo mapa conceptual de la investigación científica en ciencias de los alimentos basado en técnicas cienciométricas en España (2003-2014). // Ibersid. 11:1 (en.jun., 2017) 13-31. ISSN 1888-0967. 
Observamos ahora en la Figura 6 el mapa general anterior (Figura 2) representando en este caso la densidad. Este mapa destaca las zonas con mayor concentración de nodos con un rojo cada vez más intenso, mostrándonos así, de manera muy clara, los campos que tienen mayor cohesión interna. Nos encontramos con un mapa que destaca especialmente 8 zonas y con menor intensidad otras 6 . Si damos por hecho que cada zona destacada se corresponde con un subcampo, y sabiendo que en total tenemos
18 subcampos, en total tenemos 14 subcampos representados en el mapa, quedando 4 sin presentar, lo que indica que estos 4 no presentan gran cohesión interna entre sus palabras clave. Las áreas destacadas son indicativas de potentes frentes de investigación $y$, como decíamos antes, de temas candentes. Comparado con el mapa de densidad del caso mundial (Figura 7), se observa claramente que el del caso español es mucho más denso presentando más zonas destacadas.

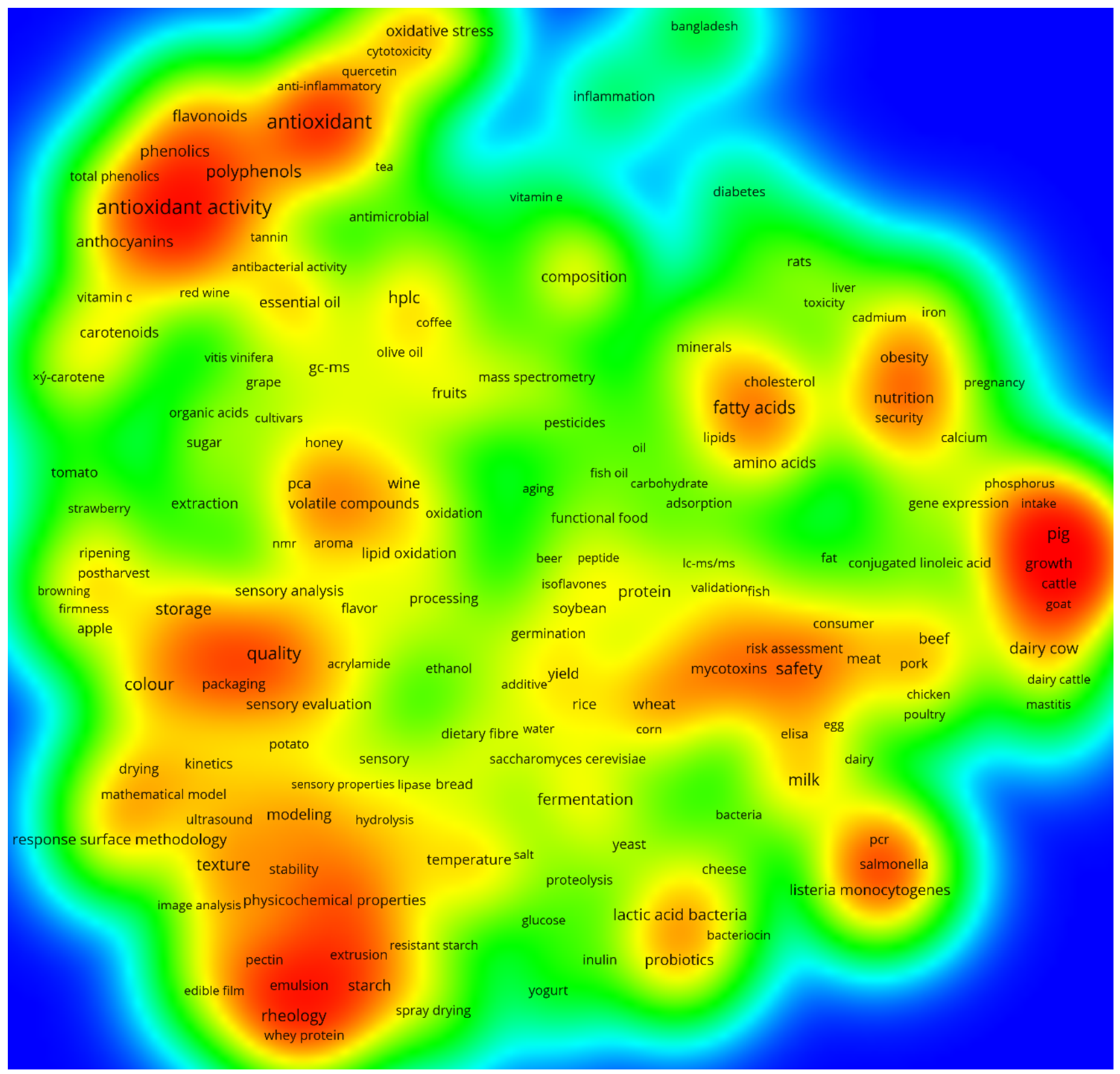

Figura 7. Vista de densidad del mapa general mundial de copalabras

En este nuevo mapa aparece representada la red de copalabras coloreada en función de la citación normalizada promedio que obtienen los documentos etiquetados con las palabras clave del mapa. Mientras el color del nodo tienda más al rojo, mayor citación tendrán sus documentos 
asociados. Siendo así, a simple vista se observa que es la zona norte, correspondiente a los "Antioxidantes en Alimentos", la que más destacada aparece en cuanto a intensidad de color mostrando ser la zona del conjunto donde hay mayor concentración de documentos muy citados. Destaca también la zona suroeste con más tonos amarillos, anaranjados y rojizos, correspondiente sobre todo a la "Seguridad Alimentaria".

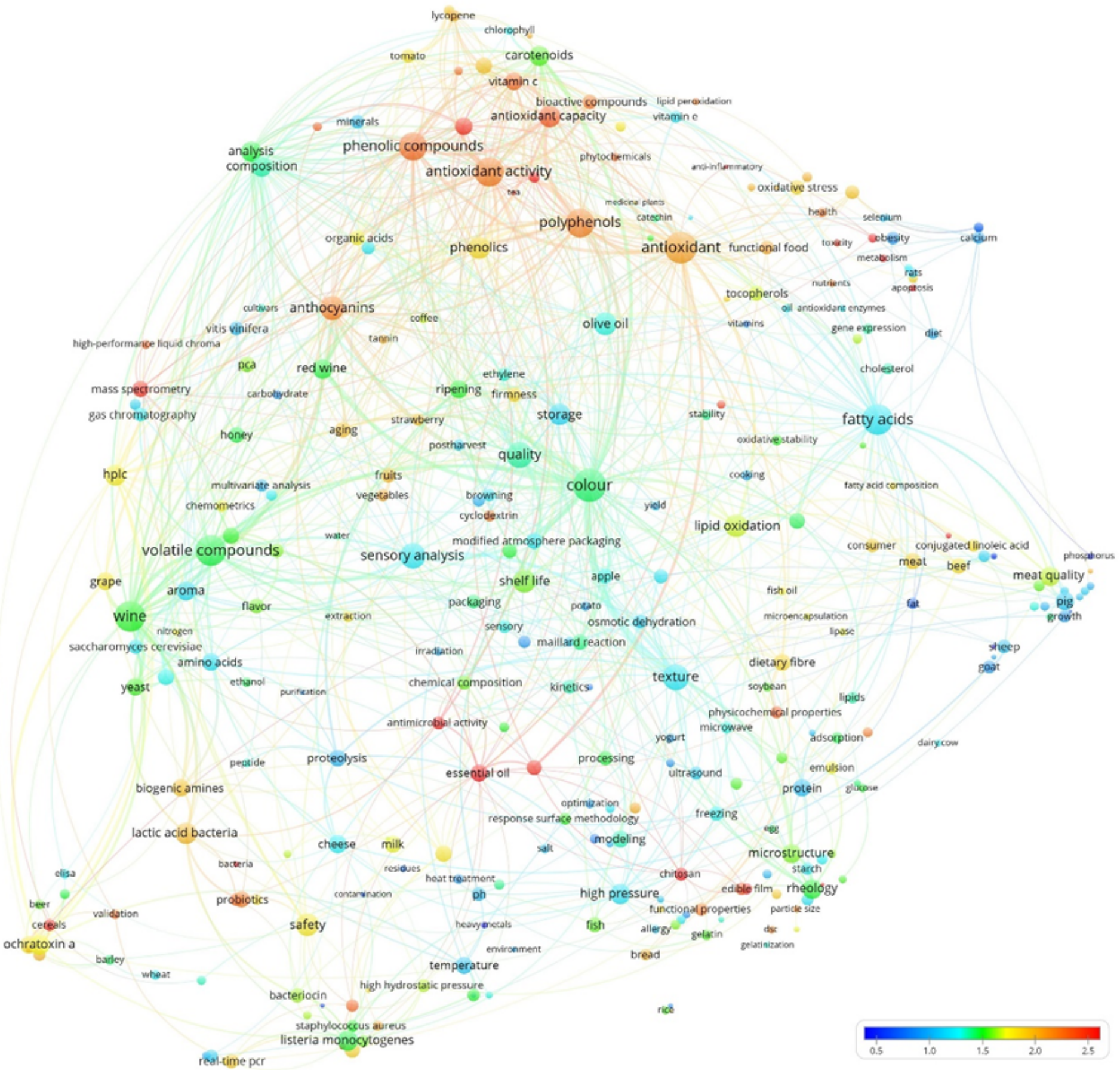

Figura 8. Vista general del mapa de copalabras donde los colores marcan la Citación Normalizada Promedio obtenida por los documentos etiquetados con dichas palabras clave. No se ha contabilizado la producción de 2014 por considerar que los datos de citación no son lo suficientemente fiables.

Aparte hay más palabras clave dispersas por el mapa con una gran citación asociada a sus documentos y, de forma general, son muy po- cos los nodos que presentan un tono azul oscuro, que son los que se identifican con los documentos menos citados cayendo por debajo de la 
media 1. Todos estos datos nos permiten confirmar que las palabras clave utilizadas para la detección de los campos poseen una gran frecuencia de aparición, estando presentes en los documentos y temas con mayor impacto científico. En la Tabla 1 que veremos más adelante, tenemos los datos concretos de citación. Si a su vez este mapa lo volvemos a colorear pero en esta ocasión en función de la citación normali- zada relativa de España con respecto al mundo, queda así (Figura 9). En este mapa se observa que casi todos los nodos superan la media 1 , esto quiere decir que la citación normalizada de España es superior, de manera relativa, a la citación normalizada del mundo, siendo los nodos que más se acercan al color rojo los que mayor diferencia positiva tienen en cuanto a impacto científico con respecto al mundo.

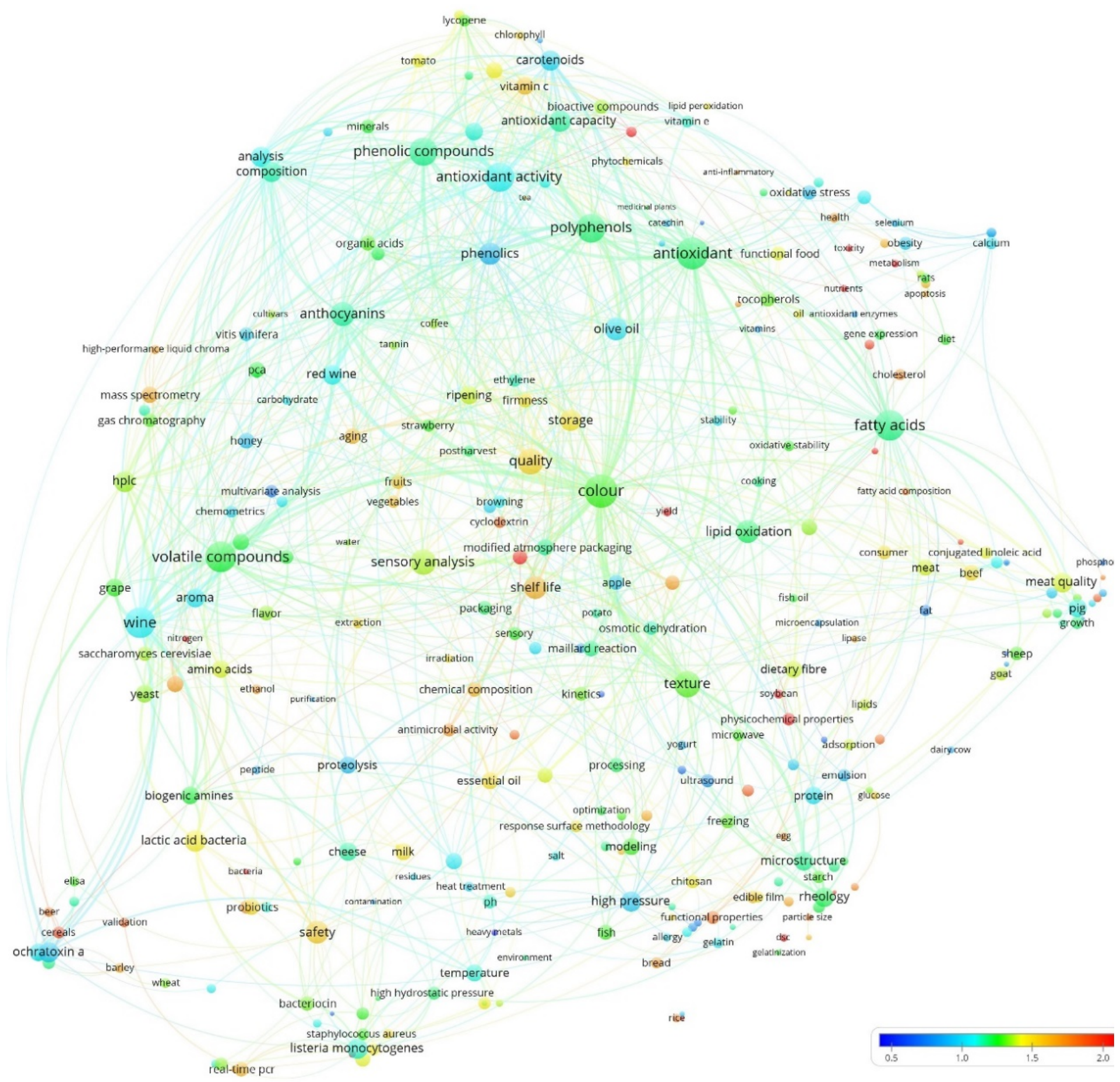

Figura 9. Vista general del mapa de copalabras donde los colores marcan la Citación Normalizada Relativa respecto al mundo 
A continuación, mostramos un resumen de los datos obtenidos para el caso español a través de una serie de indicadores presentados en dos tablas, una para los campos generales y otra para los subcampos:

\begin{tabular}{lllllllll}
\hline Campo & Ndocc & Citas & CN & Percentil & \%Exc & \%Exc1 & \%Citados & $\%$ \\
\hline 1. Composición de Alimentos y Nutrición & 2176 & 14,67 & 1,35 & 37,94 & 14,29 & 0,74 & 91,45 & 16,87 \\
\hline 2. Procesado y Modificado de Alimentos & 2061 & 15,74 & 1,52 & 35,09 & 17,90 & 1,31 & 93,89 & 15,98 \\
\hline 3. Seguridad Alimentaria & 1980 & 15,66 & 1,63 & 33,13 & 19,34 & 1,82 & 93,79 & 15,35 \\
\hline 4. Conservación de Alimentos y Vida útil & 3057 & 15,70 & 1,40 & 36,26 & 15,96 & 0,75 & 93,75 & 23,71 \\
\hline 5. Antioxidantes en Alimentos & 1615 & 18,65 & 2,00 & 28,26 & 27,93 & 2,91 & 94,80 & 12,52 \\
\hline
\end{tabular}

Tabla I. Tabla resumen de los 5 campos generales o clústeres de resolución 1

En la Tabla I presentamos el resumen de los 5 campos generales a través del dato exacto de una serie de indicadores concretos: número de documentos que contiene cada campo (Ndocc), número de citas promedio (Citas), citación normalizada $(\mathrm{CN})$, percentil promedio en el que se encuentran los documentos según las citas (dentro de cada tipo documental y año de publicación. Percentil), porcentaje de documentos excelentes dentro del $10 \%$ y del $1 \%$ más citados (\%Exc y \%Exc1), porcentaje total de documentos citados al menos una vez (\%Citados) y porcentaje de documentos con respecto al total (\%).

Podemos observar datos tan relevantes como la posición de los 5 campos por encima de la media 1 en cuanto a citación normalizada (esto también se observa muy bien en la Figura 8 antes comentada).

Otro dato importante, que además está en estrecha relación con el de la citación normalizada, es que el porcentaje de documentos excelentes que se encuentra dentro del $10 \%$ de los documentos más citados (\%Exc), supera en los 5 campos la media 10. No ocurre igual con los documentos excelentes dentro del 1\% (\%Exc1) en el cual, los campos dedicados a la "Compo- sición de alimentos y nutrición" y a la "Conservación de alimentos y vida útil" se quedan por debajo de la media 1.

El campo que se ocupa de los antioxidantes es el que mayor porcentaje de documentos excelentes tiene, superando en casi 8 puntos al segundo con mayor porcentaje en "\%Exc", que es el correspondiente a la "Seguridad alimentaria"; y en más de 1 punto al segundo con mayor porcentaje en "\%Exc1", que también vuelve a ser el campo 3 de "Seguridad alimentaria". Esto indica que el campo número5 es el que mayor impacto científico tiene de los cinco campos generales y, además, que cuenta con una gran cohesión interna y especialización. Si nos fijamos en el resto de indicadores de la tabla, comprobamos que este campo es el que mejores datos obtiene en todos ellos, excepto en el número de documentos y, como es lógico, en el porcentaje de documentos con respecto al total.

En el otro lado se encuentra el campo número 1 dedicado a la "Composición de alimentos y nutrición" con los datos más bajos en todos los indicadores de la tabla, excepto en el número de documentos y en el porcentaje de documentos con respecto al total, aunque en estos dos, tampoco es el que más tiene.

\begin{tabular}{crrrrrrrc}
\hline Subcampo & Ndocc & Citas & CN & Percentil & \%Exc & \%Exc1 & \%Citados & $\%$ \\
\hline 1.1 & 424 & 15,25 & 1,44 & 39,98 & 14,86 & 1,42 & 88,68 & 3,29 \\
\hline 1.2 & 550 & 14,35 & 1,33 & 38,40 & 13,82 & 0,91 & 90,55 & 4,26 \\
\hline 1.3 & 922 & 14,07 & 1,27 & 38,46 & 13,34 & 0,11 & 92,41 & 7,15 \\
\hline 1.4 & 527 & 15,85 & 1,50 & 33,93 & 16,89 & 0,95 & 93,55 & 4,09 \\
\hline 2.1 & 876 & 16,1 & 1,69 & 33,40 & 22,15 & 1,71 & 94,29 & 6,79 \\
\hline 2.2 & 855 & 14,81 & 1,31 & 37,06 & 13,33 & 0,58 & 93,45 & 6,63 \\
\hline 2.3 & 291 & 17,66 & 1,73 & 32,57 & 20,27 & 2,41 & 94,85 & 2,26 \\
\hline 2.4 & 241 & 14,44 & 1,31 & 38,31 & 13,69 & 0,41 & 91,29 & 1,87 \\
\hline 3.1 & 758 & 15,81 & 1,61 & 32,86 & 18,47 & 1,98 & 94,33 & 5,88 \\
\hline 3.2 & 680 & 15,39 & 1,61 & 32,78 & 19,12 & 0,74 & 93,82 & 5,27 \\
\hline
\end{tabular}




\begin{tabular}{rrrrrrrrr}
\hline 3.3 & 432 & 14,68 & 1,56 & 34,43 & 18,75 & 1,85 & 93,06 & 3,35 \\
\hline 3.4 & 277 & 17,69 & 1,94 & 31,06 & 25,63 & 5,05 & 94,58 & 2,15 \\
\hline 4.1 & 1432 & 15,97 & 1,38 & 36,12 & 15,85 & 0,70 & 95,04 & 11,10 \\
\hline 4.2 & 1406 & 15,75 & 1,47 & 35,46 & 16,71 & 0,78 & 93,31 & 10,90 \\
\hline 4.3 & 295 & 15,80 & 1,26 & 37,15 & 13,22 & 0,00 & 92,88 & 2,29 \\
\hline 4.4 & 269 & 14,67 & 1,38 & 38,75 & 16,36 & 0,74 & 90,71 & 2,09 \\
\hline 5.1 & 1394 & 19,07 & 2,08 & 27,45 & 29,99 & 3,30 & 94,62 & 10,81 \\
\hline 5.2 & 363 & 17,55 & 1,75 & 29,29 & 23,69 & 1,10 & 96,69 & 2,81 \\
\hline
\end{tabular}

Tabla II. Tabla resumen de los 18 subcampos o clústeres de resolución 2

La Tabla II presenta un resumen con los mismos datos que la Tabla I, pero en esta ocasión de los 18 subcampos específicos.

Fijándonos en la citación normalizada comprobamos que nuevamente estos 18 subcampos se encuentran por encima de la media 1. Además, si observamos la columna con los datos relativos al porcentaje de documentos excelentes que se encuentran dentro del $10 \%$ de los documentos más citados (\%Exc), también los 18 superan la media, en este caso de 10. Pero pasando ya al porcentaje de documentos excelentes que se encuentra dentro del $1 \%$ más citado, aquí no todos superan la media, es más, la mayoría de ellos están por debajo de ella e incluso uno de ellos, el 4.3 dedicado a los "Parámetros que condicionan la Conservación de Alimentos", no incluye a ninguno de los documentos asociados a él entre los documentos del $1 \%$ más citado.

Es el subcampo 5.1, dedicado a los "Antioxidantes y sus Efectos", el que mejores resultados obtiene en varios de los indicadores de la tabla: Citas, CN, Percentil, \%Exc. En Ndocc y \% es el 4.1, "Incremento de vida útil en alimentos vegetales durante la conservación. Métodos y modificaciones de calidad", el que se sitúa a la cabe- za y por último en \%Exc1 es el 3.4, dedicado a "Agentes Antimicrobianos utilizados en Alimentos. Métodos de Determinación de estos Agentes Antimicrobianos", el que mejor valor obtiene, y en \%citados lo es el subcampo 5.2, correspondiente a los "Antioxidantes Vegetales".

En cuanto a los peores resultados obtenidos, hacemos un breve repaso. El subcampo 2.4, dedicado a "Modificaciones de Alimentos durante el Procesado y Forma de determinar estos Cambios", es el que menos Ndocc y \% tiene; el 1.3 , que se corresponde con la "Composición de Alimentos", obtiene los peores valores en número de citas; el 4.3, "Parámetros que condicionan conservación de alimentos", saca los peores resultados en la $\mathrm{CN}$, en \%Exc y en \%Exc1, en este último obteniendo un porcentaje igual a 0 como señalábamos antes; por último, es el subcampo 1.1, dedicado a la "Nutrición y el Metabolismo", el que menor puntuación obtiene en Percentil y en \%citados.

Si estos datos de España, de manera relativa, los comparamos con los del mundo, podemos saber cuán relevante puede llegar a ser la investigación científica española, en CA, a nivel global (Tabla III).

\begin{tabular}{rrrrrrrrr}
\hline Campo & \%Ndocc & Citas & CN & Percentil & \%Exc & \%Exc1 & \%Citados & IET \\
\hline 1. Composición de alimentos y nutrición & 7,56 & 1,33 & 1,33 & 0,78 & 1,49 & 0,80 & 1,09 & 1,22 \\
\hline 2. Procesado y Modificado de Alimentos & 7,76 & 1,27 & 1,28 & 0,79 & 1,45 & 1,11 & 1,09 & 1,25 \\
\hline 3. Seguridad Alimentaria & 7,27 & 1,26 & 1,38 & 0,74 & 1,56 & 1,60 & 1,09 & 1,17 \\
\hline 4. Conservación de Alimentos y Vida útil & 12,10 & 1,35 & 1,33 & 0,77 & 1,57 & 0,96 & 1,10 & 1,95 \\
\hline 5. Antioxidantes en Alimentos & 9,19 & 1,17 & 1,21 & 0,75 & 1,31 & 1,02 & 1,08 & 1,48 \\
\hline Subcampo & $\%$ Ndocc & Citas & CN & Percentil & $\%$ Exc & $\%$ Exc1 & $\%$ Citados & IET \\
\hline 1.1 & 4,72 & 1,38 & 1,35 & 0,81 & 1,41 & 0,96 & 1,09 & 0,76 \\
\hline 1.2 & 6,22 & 1,38 & 1,46 & 0,77 & 1,82 & 1,75 & 1,08 & 1,00 \\
\hline 1.3 & 10,36 & 1,22 & 1,24 & 0,81 & 1,35 & 0,16 & 1,09 & 1,67 \\
\hline 1.4 & 9,82 & 1,26 & 1,33 & 0,75 & 1,47 & 0,86 & 1,08 & 1,58 \\
\hline 2.1 & 6,86 & 1,26 & 1,34 & 0,78 & 1,62 & 1,22 & 1,07 & 1,10 \\
\hline
\end{tabular}




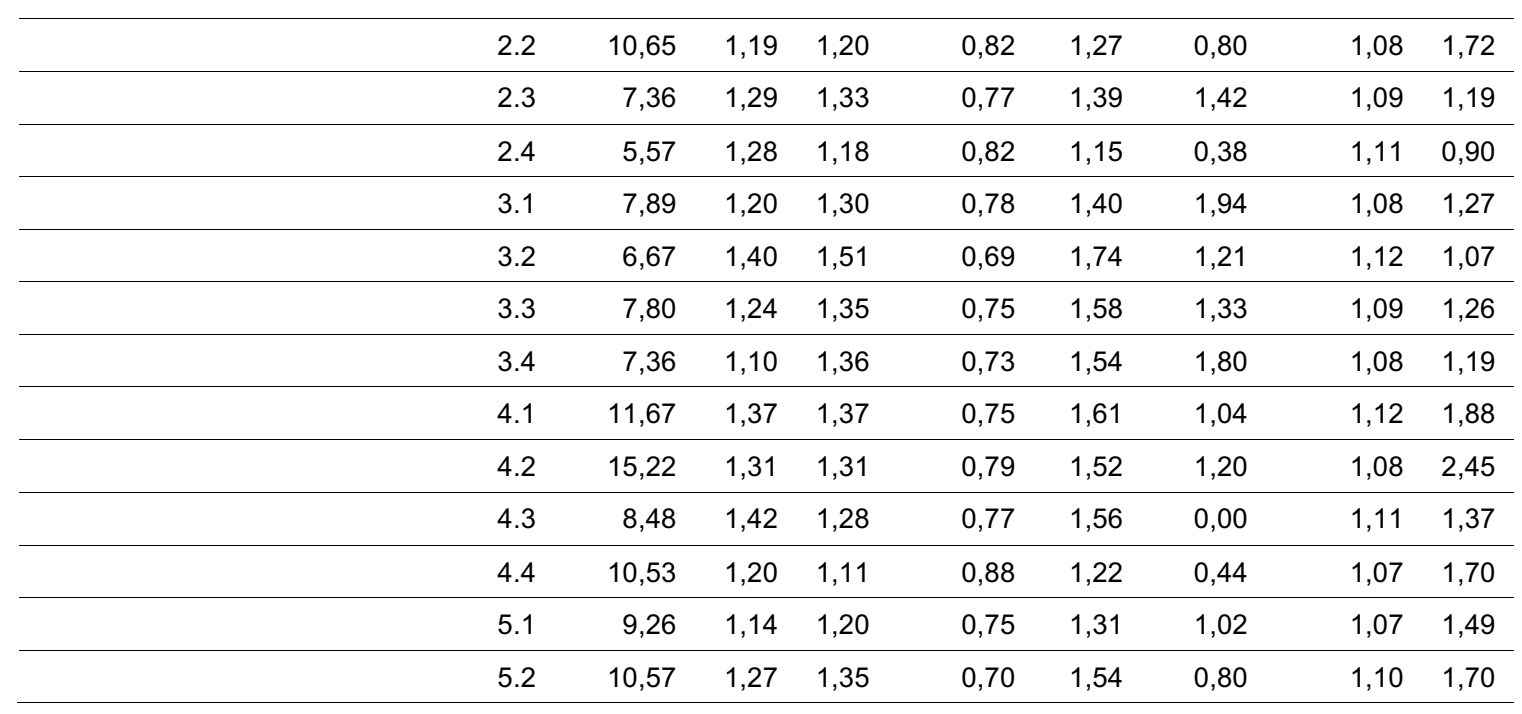

Tabla III. Tabla resumen de los campos y subcampos con los datos relativos respecto al mundo

En la primera columna de esta Tabla 3 vemos el porcentaje que supone la producción española en el conjunto del mundo, siendo el campo dedicado a la "Conservación de alimentos y vida útil" el que más aporta con un $12,10 \%$, es decir, de todo lo que se publica en el mundo sobre este tema, el 12,10\% proviene de España. Entre los subcampos, el 4.2 sobre el "Proceso de fermentación en vino y cerveza como método de conservación", es el que mayor porcentaje ostenta, un $15,22 \%$. Para el resto de indicadores se establece una media de 1 y tomándola como referencia sabemos que si el valor de un indicador es mayor que 1 quiere decir que España está superando al mundo para ese indicador concreto, si es menor, el mundo supera a España.

En la columna de citas vemos que tanto los campos como los subcampos superan en todas las ocasiones la media 1, esto quiere decir que de manera relativa España genera mayor porcentaje de citas que el conjunto mundial. Por ejemplo, en el campo 4, España genera un 35\% más de citas que el mundo. En la columna de la citación normalizada pasa igual, en todos los casos se supera la media 1 implicando el mismo significado que en la columna anterior para España. En el caso de los percentiles la cosa cambia, ya que vemos que todos los campos y subcampos se encuentran siempre por debajo de la media 1, esto se debe a que el indicador tiene sentido contrario, cuanto más citado es un trabajo en un percentil más bajo se encuentra.

En cuanto al porcentaje de documentos excelentes entre el $10 \%$ y $1 \%$ más citado vemos que siempre se supera la media 1 para el $10 \%$, pero no así para el $1 \%$ donde hay varios campos y subcampos que no llegan a la media 1 . Y en la columna de porcentaje de documentos citados al menos en una ocasión, vemos que nuevamente se supera la media 1 en todos los casos.

Por último, en la columna dedicada al índice de especialización temática (IET) nos damos cuenta que en las filas correspondientes a los 5 campos generales la media siempre es superior a 1, esto significa que estos 5 campos presentan mayor especialización temática en España que en el mundo, ya que para que fuese al revés este indicador tendría que ser inferior a 1 , algo que sucede en tan solo 2 subcampos, en el 1.1, "Nutrición y metabolismo" y en el 2.4, "Modificaciones de alimentos durante el procesado y forma de determinar estos cambios", el resto vuelven a superar al mundo, en algunos casos por mucha diferencia. Destacamos el campo dedicado a la "Conservación de alimentos y vida útil", que presenta una especialización temática superior en un $95 \%$ con respecto al mundo; y también el subcampo 4.2, "Proceso de fermentación en vino y cerveza como método de conservación", que supera al mundo en un $145 \%$.

Finalmente, en estas dos tablas (IV y $\mathrm{V}$ ) vemos el análisis de modas realizado para cada uno de los casos. En ambos casos hemos hecho el promedio de intensidad de todas las palabras clave que presentaban periodos de moda para finalmente quedarnos con las que superan ese promedio mínimo de intensidad y a partir de ellas poder comparar los 2 casos.

En ambos casos observamos que los 5 campos generales aparecen representados, pero no ocurre lo mismo con los subcampos. En el caso español no aparecen ni el 2.3, ni el 4.4, ni el 5.2; en el mundo, además de los 3 que no aparecen en el caso español, tampoco presentan periodos de moda el 2.4, el 3.4 y el 4.2. Aunque en am- 
bos casos los periodos de moda duran periodos de tiempo similares, es cierto que en el caso español encontramos palabras clave que mantienen su periodo de moda durante 4 y 5 años, algo que no ocurre en el ámbito mundial donde los máximos periodos son de 3 años.

\begin{tabular}{|c|c|c|c|c|c|}
\hline Palabra clave & $\begin{array}{l}\underset{\mathbb{D}}{\overrightarrow{0}} \\
\stackrel{0}{F}\end{array}$ & $\begin{array}{l}\stackrel{0}{\vec{\Phi}} \\
\stackrel{\Phi}{0} \\
\stackrel{0}{F}\end{array}$ & $\stackrel{\mathscr{D}}{\mathbb{D}}$ & হ⿳亠口冖几 & 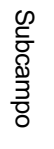 \\
\hline Zinc & 4 & 4,50 & 2003 & 2006 & 1.1 \\
\hline Obesity & 3 & 4,53 & 2013 & & 1.1 \\
\hline Calcium & 4 & 4,79 & 2003 & 2006 & 1.1 \\
\hline Inflammation & 3 & 5,81 & 2013 & & 1.1 \\
\hline Goat & 2 & 4,90 & 2009 & 2010 & 1.2 \\
\hline conjugated linoleic acid & 3 & 4,62 & 2008 & 2010 & 1.3 \\
\hline olive oil & 1 & 4,37 & 2004 & 2004 & 1.3 \\
\hline Lipids & 2 & 4,75 & 2003 & 2004 & 1.3 \\
\hline Meat & 1 & 5,12 & 2003 & 2003 & 1.4 \\
\hline consumer behaviour & 4 & 5,08 & 2012 & & 1.4 \\
\hline cyclodextrin & 1 & 5,68 & 2007 & 2007 & 2.1 \\
\hline encapsulation & 3 & 4,47 & 2013 & & 2.1 \\
\hline drying & 5 & 4,31 & 2003 & 2007 & 2.2 \\
\hline high pressure & 3 & 5,49 & 2005 & 2007 & 2.2 \\
\hline kinetics & 4 & 5,46 & 2003 & 2006 & 2.2 \\
\hline enzymes & 5 & 4,39 & 2003 & 2007 & 2.4 \\
\hline foodborne pathogen & 1 & 4,76 & 2008 & 2008 & 3.1 \\
\hline real-time pcr & 2 & 4,42 & 2011 & 2012 & 3.1 \\
\hline biofilm & 4 & 5,44 & 2012 & & 3.1 \\
\hline Ic-ms/ms & 4 & 7,39 & 2012 & & 3.2 \\
\hline composition & 2 & 5,04 & 2010 & 2011 & 3.3 \\
\hline analysis & 3 & 5,25 & 2010 & 2012 & 3.3 \\
\hline gc-ms & 2 & 5,62 & 2011 & 2012 & 3.4 \\
\hline storage & 3 & 6,67 & 2003 & 2005 & 4.1 \\
\hline ethylene & 5 & 5,73 & 2003 & 2007 & 4.1 \\
\hline ripening & 1 & 4,26 & 2003 & 2003 & 4.1 \\
\hline proteolysis & 4 & 9,64 & 2003 & 2006 & 4.1 \\
\hline Beer & 5 & 4,54 & 2011 & & 4.2 \\
\hline lactic acid bacteria & 1 & 4,06 & 2008 & 2008 & 4.2 \\
\hline fermentation & 1 & 4,58 & 2008 & 2008 & 4.2 \\
\hline $\mathrm{Ph}$ & 6 & 7,97 & 2003 & 2008 & 4.3 \\
\hline bioactive compounds & 3 & 6,09 & 2013 & & 5.1 \\
\hline phytochemicals & 3 & 4,47 & 2013 & & 5.1 \\
\hline oxidative stress & 2 & 5,44 & 2014 & & 5.1 \\
\hline phenolic compounds & 3 & 7,98 & 2013 & & 5.1 \\
\hline
\end{tabular}

Tabla IV. Modas de palabras clave con intensidad superior a 4 (España)

\begin{tabular}{|c|c|c|c|c|c|}
\hline Palabra clave & 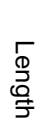 & 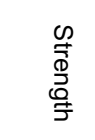 & $\stackrel{\mathscr{D}}{\stackrel{D}{\rightleftharpoons}}$ & 罟 & 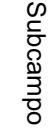 \\
\hline Inflammation & 4 & 29,93 & 2012 & & 1.1 \\
\hline Security & 3 & 27,20 & 2013 & & 1.1 \\
\hline Obesity & 2 & 18,05 & 2014 & & 1.1 \\
\hline Sustainability & 3 & 13,36 & 2013 & & 1.1 \\
\hline Pig & 4 & 81,08 & 2003 & 2006 & 1.2 \\
\hline Sheep & 2 & 29,16 & 2003 & 2004 & 1.2 \\
\hline Growth & 3 & 25,77 & 2003 & 2005 & 1.2 \\
\hline beef cattle & 2 & 12,84 & 2003 & 2004 & 1.2 \\
\hline Digestibility & 1 & 12,50 & 2004 & 2004 & 1.2 \\
\hline Carcass & 2 & 11,98 & 2003 & 2004 & 1.2 \\
\hline Fat & 3 & 16,73 & 2003 & 2005 & 1.3 \\
\hline conjugated linoleic acid & 3 & 14,48 & 2003 & 2005 & 1.3 \\
\hline Beef & 2 & 28,72 & 2003 & 2004 & 1.4 \\
\hline Tenderness & 2 & 13,36 & 2003 & 2004 & 1.4 \\
\hline Pork & 2 & 12,42 & 2003 & 2004 & 1.4 \\
\hline Cyclodextrin & 2 & 30,94 & 2006 & 2007 & 2.1 \\
\hline Encapsulation & 3 & 14,31 & 2013 & & 2.1 \\
\hline mathematical model & 2 & 17,21 & 2014 & & 2.2 \\
\hline high pressure & 4 & 10,76 & 2003 & 2006 & 2.2 \\
\hline Salmonella & 1 & 10,92 & 2012 & 2012 & 3.1 \\
\hline milk production & 2 & 10,18 & 2003 & 2004 & 3.1 \\
\hline Ic-ms $/ \mathrm{ms}$ & 3 & 24,44 & 2013 & & 3.2 \\
\hline Composition & 3 & 29,19 & 2009 & 2011 & 3.3 \\
\hline Analysis & 2 & 22,19 & 2010 & 2011 & 3.3 \\
\hline Proteolysis & 2 & 26,60 & 2003 & 2004 & 4.1 \\
\hline Irradiation & 2 & 15,43 & 2003 & 2004 & 4.1 \\
\hline Proteolysis & 1 & 38,12 & 2006 & 2006 & 4.1 \\
\hline Irradiation & 1 & 20,58 & 2007 & 2007 & 4.1 \\
\hline $\mathrm{Ph}$ & 1 & 14,76 & 2003 & 2003 & 4.3 \\
\hline oxidative stress & 3 & 37,81 & 2013 & & 5.1 \\
\hline bioactive compounds & 3 & 24,07 & 2013 & & 5.1 \\
\hline anti-inflammatory & 4 & 14,70 & 2012 & & 5.1 \\
\hline
\end{tabular}

Tabla V. Modas de palabras clave con intensidad superior a 10 (Mundo)

Por otra parte, aunque hay una cantidad considerable de palabras clave que presentan periodos de moda en los 2 casos, la mayoría solo aparece en uno de los 2 .

\section{Conclusiones}

España es sin duda una potencia en lo que a investigación en CA se refiere y no tanto por su volumen de producción científica, que también, 
sino más bien por la calidad de ésta. En los resultados de este trabajo se observa como en numerosas ocasiones y para diferentes aspectos de la investigación en CA, España supera al conjunto mundial.

La evolución de la producción española en la investigación referente a las CA crece año a año además en todos los campos generales, exceptuando una leve caída ocurrida en 2010. De manera relativa al mundo observamos que la producción se mantiene de manera estable sin crecer ni decrecer de manera destacable, solo el campo 4, "Conservación de alimentos y vida útil", desciende durante el periodo estudiado de manera más pronunciada, pero junto con el 5 , "Antioxidantes en alimentos", son los únicos que se mantienen por encima de la producción mundial. Los otros 3 campos generales aunque están por debajo de la media se mantienen muy cerca de ésta produciendo a un nivel similar al conjunto mundial.

En cuanto a las relaciones que mantienen los 5 campos generales entre sí, cabe destacar en primer lugar que sus nodos se relacionan, por regla general, de mejor manera con nodos de su mismo campo. Pero salvando este dato que puede parecer obvio, observamos en los resultados como en el caso español las relaciones mantenidas entre áreas son diferentes a las del caso mundial. Todas estas diferencias en las relaciones de un caso y otro, vienen a decirnos que aunque la investigación internacional en CA puede estar sujeta a una misma estructura temática, según el caso específico estudiado, las relaciones estructurales pueden variar y formarse de distinta manera. Recordemos que las relaciones, son consecuencia de los trabajos que incluyen palabras claves pertenecientes a ambas áreas.

La cohesión de los 5 campos generales es diferente en cada uno de ellos. Los campos 4, "Conservación de alimentos y vida útil", y 5, "Antioxidantes en alimentos", son los que presentan mayor cohesión interna, el 5 algo más, y también el número 1, "Composición de alimentos y nutrición". El 2, "Procesado y modificado de alimentos", y 3 , "Seguridad alimentaria", están menos cohesionados especialmente el número 3.

Aun así, si comparamos con el mundo el mapa de copalabras construido para España, se ve claramente que aparece mucho más cohesionado, siendo esto indicativo de que en general la investigación está más especializada y posiblemente goza de una mejor organización de la misma, algo que parece lógico ya que el territo- rio analizado es mucho menor que el mundo en general.

En lo referente a la centralidad, al contrario que en el mundo, donde ningún campo destacaba por su centralidad, en España sí que lo hace el número 4, "Conservación de alimentos y vida útil", claramente. $Y$ en el tema de la especialización nuevamente son los campos 4 y 5 los más especializados, superando el 4 en un $95 \%$ de especialización temática al mundo, al igual que el resto de campos también lo superan aunque con porcentajes menores pero aun así muy importantes.

Si hablamos de impacto científico, España sigue estando destacada conforme al mundo y vuelve a superar al conjunto global en lo que a citación normalizada se refiere y además en todos los campos. Esto también se nota en el gran porcentaje de documentos excelentes que coloca entre el $10 \%$ y el $1 \%$ más citado.

Por último, al hablar de las modas vemos que estas afectan a los 5 campos generales, ya que todos tienen alguna de sus palabras clave con periodos de moda destacados. Algo que no ocurre igual en los subcampos, donde no todos cuentan con palabras clave con periodos de moda. Eso sí, si comparamos con el mundo, España presenta más subcampos que el mundo con periodos de moda y además se mantienen activados durante periodos de tiempo más amplios.

La mayoría de las palabras clave aparecen en uno u otro caso, no suelen coincidir en los dos, por ello podemos afirmar que las modas de términos no coinciden en el ámbito español y mundial.

En definitiva, España debe seguir trabajando en la dirección actual ya que se sitúa a la cabeza de la investigación en CA, superando de manera relativa al conjunto mundial en un gran número de indicadores.

\section{Reconocimientos}

Este trabajo ha sido financiado por la Consejería de Educación Ciencia \& Tecnología y el Fondo Social Europeo como parte de la ayuda a grupos GR15024, y por el Plan Nacional de Investigación Científica, Desarrollo e Innovación Tecnológica 2012-2015 y los Fondos FEDER como parte del proyecto CSO2013-40530-R. También, queremos agradecer a Juan José Córdoba Ramos su ayuda en la etiquetación de la estructura de clústeres y sus comentarios respecto a los resultados.

\section{Referencias}

Blázquez-Ruiz, Jesús; Guerrero-Bote, Vicente P.; MoyaAnegón, Félix (2016). New Scientometric-Based Knowledge Map of Food Science Research (2003 to 
2014). // Comprehensive Reviews in Food Science and Food Safety. 15:6 (October 2016) 1040-1055.

Bornmann, Lutz; Moya-Anegón, Félix; Leydesdorff, Loet (2012). The new excellence indicator in the World Report of the SCImago Institutions Rankings 2011. // Journal of Informetrics. 6:2 (April 2012) 333-335.

Chinchilla-Rodríguez, Zaida; Olmeda-Gómez, Carlos (2010). Producción y colaboración científica en agroalimentación. // Sanz-Menéndez, Luis; Cruz-Castro, Laura (2010). Análisis Sobre Ciencia E Innovación En España. Madrid: FECYT, 2010. 978-84-693-6286-0. 366-399.

Damerau, Fred J. (1964). A technique for computer detection and correction of spelling errors. /I Communications of the ACM. 7:3 (March 1964) 171-176.

Falagas, Matthew E.; Pitsouni, Eleni I.; Malietzis, George A.; Pappas, Georgios (2008). Comparison of PubMed, Scopus, Web of Science, and Google Scholar: strengths and weaknesses. // The FASEB Journal. 22:2 (February 2008) 338-342.

Gerland, Patrick; Raftery, Adrian E.; Sevcikova, Hana; Li, Nan; Gu, Danan; Spoorenberg, Thomas; Alkema, Leontine; Fosdick, Bailey K.; Chunn, Jennifer; Lalic, Nevena; Bay, Guiomar; Buettner, Thomas; Heilig, Gerhard K.; Wilmoth, John (2014). World population stabilization unlikely this century. // Science. 346:6206 (October 2014) 234-237.

Granda-Orive, José I.; Alonso-Arroyo, Adolfo; García-Río, Francisco; Solano-Reina, Segismundo; Jiménez-Ruiz, Carlos A.; Aleixandre-Benavent, Rafael (2013). Ciertas ventajas de Scopus sobre Web of Science en un análisis bibliométrico sobre tabaquismo. // Revista Española de Documentación Científica. 36:2 (Abril-Junio 2013) e011.

Guerrero-Bote, Vicente P.; Moya-Anegón, Félix (2013). Producción científica en Food Science (Scopus, 20032013).

Guerrero-Bote, Vicente P; Olmeda-Gómez, Carlos; MoyaAnegón, Félix (2016a). Atlas of scientific institutions in food science (Scopus, 2003-2013). // LWT - Food Science and Technology. 67 (April 2016) 133-142.

Guerrero-Bote, Vicente P; Olmeda-Gómez, Carlos; MoyaAnegón, Félix (2016b). La Ciencia De Los Alimentos Georreferenciada. Aproximación Bibliométrica a Nivel Institucional. // El Profesional de La Información. 25:1 (enero-febrero 2016) 25-34

IFT (2016). About Food Science and Technology. // Institute of food technologists. http://www.ift.org/knowledgecenter/learn-about-food-science/food-facts/about-fs-andt.aspx (2016-04-13).

Kleinberg, Jon. (2003). Bursty and Hierarchical Structure in Streams. // Data Mining and Knowledge Discovery. 7:4 (October 2003) 373-397.
Levenshtein, Vladimir I. (1966). Binary codes capable of correcting deletions, insertions, and reversals. // Soviet Physics Doklady. 10:8 (February 1966) 707-710.

Mingers, John; Leydesdorff, Loet (2015). A Review of Theory and Practice in Scientometrics. // European Journal of Operational Research. 246:1 (October 2015) 1-19.

Moya-Anegón, Félix; Vargas-Quesada, Benjamín; Chinchilla-Rodríguez, Zaida; Herrero-Solana, Víctor; Corera-Álvarez, Elena (2006). Análisis de la cobertura de la base de datos Scopus. // El Profesional de La Información. 15:2 (marzo-abril 2006) 144-145.

Newman, Mark; Girvan, Michelle (2004). Finding and evaluating community structure in networks. // Physical Review E. 69:2 (February 2004) 026113.

Noack, Andreas (2007). Energy Models for Graph Clustering. II Journal of Graph Algorithms and Applications. 11:2 (February 2007) 453-480.

Noack, Andreas (2009). Modularity clustering is forcedirected layout. // Physical Review E. 79:2 (February 2009) 026102

Rehn, Catharina; Kronman, Ulf (2008). Bibliometric handbook for Karolinska Institutet. Hudinge: Karolinska Institutet, 2008.

Romo-Fernández, Luz M; Guerrero-Bote, Vicente P.; MoyaAnegón, Félix (2013). Co-word based thematic analysis of renewable energy (1990-2010). // Scientometrics. 97:3 (December 2013) 743-765.

Van Eck, N.J. (2011). Methodological Advances in Bibliometric Mapping of Science. ERIM PhD Series Research in Management, 247. ISBN 978-90-5892-2915.

Van-Eck, Nees J; Waltman, Ludo (2007). VOS: a new method for visualizing similarities between objects. // Decker, Reinhold; Lens, Hanz J (2007). Advances in data analysis: Proceedings of the 30th annual con-ference of the gesellschaft für klassifikation e.V., freie Universität Berlin, march 8-10, 2006. Berlin: Springer Science \& Business Media, 2007. 978-3-540-70980-0. 299-306.

Waltman, Ludo; Van-Eck, Nees J; Noyons, Ed CM (2010). A unified approach to mapping and clustering of bibliometric networks. // Journal of Informetrics. 4:4 (October 2010) 629-635.

Wiley-Blackwell (2015). The 2015 Revision of the UN's World Population Projections. // Population and development review. 41:3 (September 2015) 557-561.

Enviado: 2017-01-24. Segunda versión: 2017-04-29. Aceptado: 2017-04-29. 
